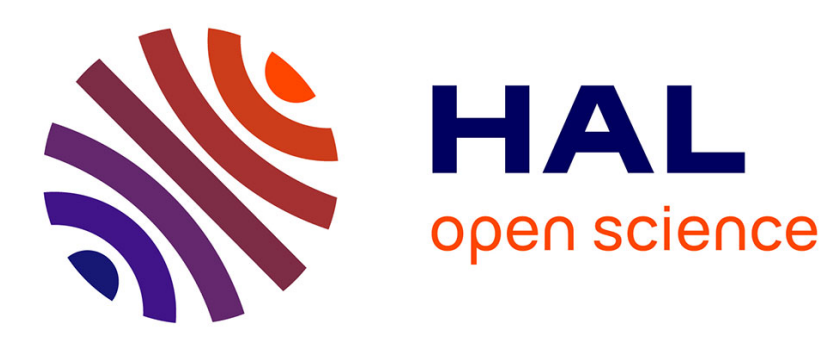

\title{
Voix, point de vue... ou comment pêcher le dialogisme à la métaphore...
}

Jacques Bres, Aleksandra Nowakowska

\section{To cite this version:}

Jacques Bres, Aleksandra Nowakowska. Voix, point de vue... ou comment pêcher le dialogisme à la métaphore.... Les cahiers de praxématique, 2008, 49, pp.103-132. hal-00435161

\section{HAL Id: hal-00435161 \\ https://hal.science/hal-00435161}

Submitted on 4 Jul 2018

HAL is a multi-disciplinary open access archive for the deposit and dissemination of scientific research documents, whether they are published or not. The documents may come from teaching and research institutions in France or abroad, or from public or private research centers.
L'archive ouverte pluridisciplinaire HAL, est destinée au dépôt et à la diffusion de documents scientifiques de niveau recherche, publiés ou non, émanant des établissements d'enseignement et de recherche français ou étrangers, des laboratoires publics ou privés. 


\author{
Jacques Bres, Aleksandra Nowakowska \\ Praxiling, UMR 5267 CNRS -Montpellier III \\ jacques.bres@univ-montp3.fr \\ aleksandra.nowakowska@univ-montp3.fr
}

\title{
Voix, point de vue... ou comment pêcher le dialogisme à la métaphore...
}

\section{Introduction}

La notion de voix, dans le champ des analyses linguistiques qui depuis une trentaine d'années s'attachent à décrire le dialogisme - ou la polyphonie ${ }^{1}$ - de l'énoncé et du discours, apparaît le plus souvent comme une évidence non questionnée, depuis les textes fondateurs de Bakhtine jusqu'aux travaux contemporains dans le domaine. Prenons un seul exemple : le récent ouvrage coordonné par L. Perrin (2006) et composé de dix-huit articles, alors même qu'il contient ladite notion dans son titre - Le sens et ses voix - ne lui consacre aucun travail spécifique $^{2}$. Confirme cela le fait que, si l'on considère les deux dictionnaires d'analyse du discours, Charaudeau et Maingueneau (2002) et Détrie, Siblot et Verine (2001), voix ne dispose pas d'entrée dans le premier ; dispose bien d'une entrée dans le second... qui se réalise sous la forme non d'un article, mais d'un renvoi à l'entrée Dialogisme. Et cette dernière notion s'y voit ainsi définie : «Capacité de l'énoncé à faire entendre, outre la voix de l'énonciateur, une ou plusieurs autres voix qui le feuillettent énonciativement» (p.83) (les italiques sont nôtres ; et il en sera de même dans les citations qui suivent). Comme si la notion de voix, qui permet de définir le dialogisme, n'avait elle-même aucunement besoin de définition, du fait de sa transparence... Ce qui est d'autant plus notable que la notion de point de vue, avec laquelle voix est en relation de glose ou de concurrence suivant les théories nous allons le voir, semble mieux lotie, au moins dans les deux dictionnaires mentionnés : elle dispose d'une entrée, suivie d'un article dans chacun des deux ouvrages... Deux poids, deux mesures... Le présent article entend questionner l'évidence du terme de voix, notamment en le mettant en relation avec son doublon, ou son rival, celui de point de vue. Pour cela, notre étude partira de l'analyse des textes russes de Bakhtine, pour examiner ensuite les approches polyphoniques de la voix et du point de vue, avant d'étudier la place et le fonctionnement de voix dans notre propre approche dialogique ${ }^{3}$.

\section{Bakhtine}

Pour étudier la notion de voix (golos) chez Bakhtine, nous nous sommes penchés sur trois textes, dans les versions russe et française : (a) l'article "Slovo v romane ", écrit en 19341935, publié en France sous le titre “ Du discours romanesque ”, dans Esthétique et théorie du roman (1978, désormais DDR) ; (b) l'article " Problema rechevyx zhanrov", écrit en 1952-

\footnotetext{
${ }^{1}$ Pour Bakhtine le dialogisme, principe qui gouverne toute pratique humaine, consiste, au niveau langagier, en l'orientation de tout discours vers d'autres discours avec lesquels il ne peut manquer d'interagir, et se manifeste comme pluralité de voix. La polyphonie est l'utilisation romanesque du dialogisme de l'énoncé quotidien. Mais à la suite de Ducrot, certains linguistes parlent de polyphonie pour appréhender l’hétérogénéité énonciative de l’énoncé quotidien.

${ }^{2}$ Seule L. Rosier (2006) lui accorde deux pages (202-203).

${ }^{3}$ Pour une synthèse, cf. Bres et Nowakowska 2005.
} 
1953, publié en France sous le titre " Les genres du discours " dans Esthétique de la création verbale (1984, désormais GD); et (c) le texte Problemy poetiki Dostoevskogo (1963), réécriture du texte de 1929, Problemy tvorchestva Dostoevskogo (Problèmes de la poétique de Dostoïevski (1970, désormais PPD).

La première remarque sera d'ordre quantitatif : il apparaît que le terme de golos (voix), et plus largement les mots liés à la vocalité, sont totalement absents de GD, le texte le plus linguistique des trois, qui use seulement de deux termes : slovo (mot) et rech' (énoncé) ${ }^{4}$.

Golos est par contre très utilisé dans les deux autres textes. En outre dans DDR, on trouve, dans la traduction française, des termes qui relèvent de la vocalité, mais qui procèdent de termes russes qui n'ont pas forcément ce type de signification : c'est le cas de raznorechie 5 traduit, entre autres, par plurilinguisme, plurivocalité ou polyphonie, et de raznogolosnitza (dissonance, divergence des points de vue) traduit, entre autres, par plurivocité.

Plusieurs questions se posent alors: quelle est la signification attribuée à golos chez Bakhtine ? Comment expliquer les différences tant quantitatives que qualitatives entre les textes ? Pourquoi ces approximations dans la traduction?

Afin de répondre à ce questionnement, nous proposons, dans un premier temps, l'analyse sémantique de golos (voix), suivie ensuite de l'étude des problèmes traductologiques liés à l'apparition en français d'un vaste champ lexical de la vocalité là où, en russe, il est plutôt question de divergence de points de vue...

\subsection{Etude sémantique de voix (golos)}

L’approche sémantique de golos révèle trois principales dimensions de la vocalité : (i) la dimension corporelle : la voix en tant que caractéristique physique de l'être humain, (ii) la dimension discursive : la voix en tant que discours (sens qui peut être exprimé également par rech') ; et enfin (iii) la dimension narratologique : la voix en tant que terme de sémiotique narrative (que l'on peut mettre en relation avec plusieurs autres termes russes mnogogolost', raznogolost', raznorechovost').

\section{(i) La dimension corporelle}

La dimension corporelle peut être appréhendée, entre autres, à partir des citations suivantes :

(1) Cherez eto voploszczennoe konkretnoe soznanie v zivom golose tzel'novo cheloveka (PPD, p.10)

C'est par l'intermédiaire de cette conscience concrète incarnée dans LA VOIX VIVANTE D'UN ÊTRE GLOBAL que la logique se relie. (PPD, p.14)

(2) Kazdoe mnenie u nego dejstvitel'no stanovitsja zivym suszczestvom i neotreszimo ot voploszczennogo czeloveceskogo golosa (PPD, p. 19).

chaque opinion devient effectivement chez lui un être vivant et elle est inséparable d'une voix humaine incarnée. (PPD, p. 23)

Dans ce type d'occurrences, golos est systématiquement déterminé par un adjectif qui renvoie à la dimension physiologique de la phonation : voix humaine incarnée, voix vivante d'un être global. La notion possède, dans ce cas, le sens qui lui est traditionnellement attribué en langue : les sons émis par les organes de la phonation au cours de la production de la parole par un sujet, la signature vocale du sujet parlant. La voix est cette matérialité nécessaire à l’expression de la pensée, incarnée dans un corps, qui permet la communication humaine.

\footnotetext{
${ }^{4}$ Le mot rech' peut vouloir dire discours, langue, langage, énoncé, parole, flux verbal, production de la parole, ce qui génère de nombreuses difficultés d'interprétation et de traduction du texte bakhtinien.

${ }^{5}$ Dans Nowakowska 2005, est étudiée plus précisément la traduction de raznorechie par polyphonie en français.
} 


\section{(ii) La dimension discursive}

La dimension discursive apparaît, entre autres, dans les occurrences suivantes :

(3) dialogicheskoe otnoszenija mogut pronikat' vnutr' vyskazyvanija, daze vnutr' otdel'nogo slova, esli v nem dialogicheski stalkivajutsja dva golosa (mikrodialog, o kotorom nam uze prixodilos' govorit'). (PPD, p. 214)

les rapports de dialogue [l'adjectif dialogique est utilisé dans le texte source : les rapports dialogiques] peuvent pénétrer à l'intérieur d'un énoncé, même à l'intérieur d'un mot séparé, si deux voix s'y rencontrent dans un dialogue (micro-dialogue dont nous avons déjà eu l'occasion de parler). (PPD, p. 214).

(4) skaz vvoditsja imenno radi chuzogo golosa, golosa sotzial'no opredelennogo, prinosjaszczego s soboj rjad tochek zrenija i otzenok, kotorye imenno i nuzny avtoru. (PPD, p. 222)

le récit parlé est introduit précisément en vue de la voix de l'autre, d'une voix socialement déterminée, apportant avec elle un certain nombre de points de vue et de jugements, lesquels sont précisément nécessaires à l'auteur. (PPD, p. 223).

Le mot golos produit ici le sens de "discours », "énoncé » (et est l'équivalent de rech', slovo), notamment pour désigner le discours de l'autre. On pourrait dire que Bakhtine utilise golos, "voix », métaphoriquement. Ce serait juste, à condition d'ajouter qu'il accorde à la discursivité une dimension vocale ; et que c'est cette dimension vocale des discours qui apparaît dans sa conception du dialogisme : les voix en interaction correspondent au dialogue interne des différents discours que rencontre un énonciateur et qui construisent sa parole.

\section{(iii) La dimension narratologique}

Bakhtine use enfin du terme de golos dans un sens narratologique, principalement dans la description du roman polyphonique de Dostoïevski :

(5) Takim obrazom, uze v nachale romana zazvuchali vse veduszczie golosa bol'szogo dialoga. Eti golosa ne zamknuty i ne gluxi drug $\mathrm{k}$ drugu. Oni vse vremja slyszat drug druga, pereklikajutsja ivzaimo otrazajutsja drug $\mathrm{k}$ druge (v mikrodialogax osobenno). I vne etogo dialoga « protivoborstvuszczix pravd » ne osuszczestvljaetsja ni odni suszczestvennyj postupok, ni odna suszczestvennaja mysl’ veduszczix geroev (PPD, p. 88)

Ainsi, dès le début du roman, entend-on toutes les principales voix du grand dialogue. Ces voix ne sont pas fermées ni sourdes les unes aux autres. Elles ne cessent de se percevoir les unes les autres, de se répondre, de se refléter mutuellement les unes les autres (particulièrement dans les microdialogues). Et hors de ce dialogue de "vérité en conflit» il ne se réalise pas une seule action essentielle, une seule pensée essentielle des héros principaux. (PPD, p. 90).

(6) Raznorechie, vvodimoe $v$ roman,- eto chuzaja rech' na chuzom jazykie, sluzaszczaja prelomlennomu vyrazeniju avtorskix intentzij. Slovo takoj rechi - osoboe dvugolosoe slovo. Ono sluzit srazu dvum govorjaszczim i vyrazaet odnovremenno dve razlichnyx intentzii :prjamujuintentziju govorjaszczego personaza i prelomlennuju avtorskuju. V takom slove dva golosa, dva smysla i dve ekspressii. Pritom eti dva golosa dialogicheski sootneseny. (DDR, p.137)

Le polylinguisme introduit dans le roman, c'est le discours d'autrui dans le langage d'autrui (...). Ce discours offre la singularité d'être bivocal. Il sert simultanément à deux locuteurs et exprime deux intentions différentes : celle - directe - du personnage qui parle, et celle - réfractée - de l'autre. Pareil discours contient deux voix, deux sens, deux expressions. En outre, les deux voix sont dialogiquement corrélatées. (DDR, p. 145).

La notion de voix permet à Bakhtine de définir les caractéristiques du roman polyphonique : dans ce type de texte, les voix sont multiples, diverses et égales. Le terme, dans ce cas, recouvre, par delà la dimension narratologique, les dimensions corporelle (voix incarnée) et 
discursive (discours produit). Dans l'occurrence (5) p. ex., la voix est indissociable, d'une part, de l'aspect phonique: les voix retentissent (zvuchat traduit par entend-on) dans le dialogue, elles ne sont pas sourdes (gluxi) les unes aux autres, elles s'écoutent (slyszat traduit par percevoir) ; et, d'autre part, de l'aspect discursif : les voix ne sont pas fermées (zamknuty) les unes aux autres, se répondent, se reflètent (otrazajutsja).

Nous avons noté au début de notre étude que golos apparaissait seulement dans les deux textes consacrés à l'écriture romanesque (DDR et PPD), et qu'il était absent de l'étude plus linguistique que constitue GD : comme si, pour Bakhtine, ce terme n'avait toute sa pertinence que pour l'écriture romanesque ; et qu'il n’était pas nécessaire à la description du dialogisme de l'énoncé quotidien.

L'absence de golos dans GD reste cependant problématique pour nous, dans la mesure où la notion de voix chez Bakhtine est liée à l'importance du dialogue (dans un sens large).Et que le dialogue est à la base de la notion de dialogisme, centrale dans GD.

\subsection{Problèmes de traduction : tentative d'explication}

L’interprétation de la vocalité chez Bakhtine a donné lieu à des approches très diverses comme celles de Ducrot, de Perrin ou de la praxématique, présentées dans la seconde partie de l'article. Ces approches s'élaborent le plus souvent à partir des traductions françaises qui présentent un flou terminologique et conceptuel lié plus particulièrement au fonctionnement heuristique de nombreuses notions chez Bakhtine. Si le terme golos est systématiquement traduit par voix en français, il apparaît que les termes de raznorechie, raznorech'vost', raznogolosnitza, qui en russe n'ont pas de rapport direct avec la voix, sont rendus en français, tantôt par un terme dérivé de voix : plurivocalité, tantôt par les termes de plurilinguisme ou de polylinguisme.

Ainsi comment expliquer le fait que, dans DDR, le terme plurivocalité traduise :

- tantôt l'adjectif raznorechivyj (discordant, divergeant, contradictoire) dérivé du nom raznorech'vost' (divergence, discordance) :

(7) Problemy stilistiki romana neizbezno privodjat k neobxodimosti kosnut'sja rjada printzipial'nyx voprosov filosofii slova svjazannyx (...) s zizn'ju i povedeniem slova v raznorechivom i raznojazychnom mire (DDR, p. 88)

Les problèmes de la stylistique du roman nous amènent inévitablement à aborder une série de questions radicales concernant la philosophie du discours, liées à (...) la vie et le comportement du discours dans un monde de plurivocalité et de plurilinguisme. (DDR, p. 98)

- tantôt raznorechie ${ }^{6}$ (traduit aussi par plurilinguisme) :

(8) Prozaik-romanist (...) idet soverszenno inym putem. On prinimaet raznorechie i raznojazychie (...) jazyka (DDR, p. 111)

Le prosateur-romancier (...) emprunte un chemin tout différent. Il accueille le plurilinguisme et la plurivocalité (...) du langage. (DDR, p. 119)

- tantôt raznogolosoe ou raznogolosnitza (divergence d'opinions, discordance) :

(9) Roman kak tzeloje - eto mnogostilnoe, raznorechivoe, raznogolosoe javlenie. (DDR, p. 75)

Le roman pris comme tout, c’est un phénomène pluristylistique, plurilingual, plurivocal. (DDR, p. 87)

\footnotetext{
${ }^{6}$ Le mot raznorechie, qui n'existe pas en russe standard, est composé d’un préfixe razno (signifiant d'une manière différente, différemment / raznoe divers) et de la racine rechie, rech' (discours, parole, dit, langue, langage, locution).
} 
(10) Roman- eto xudorzestvenno organizovannoe sotzialnoe raznorechie, inogda raznojazychie, i individualnaja raznoglosnitza. (DDR, p. 76).

Le roman c'est la diversité sociale de langages, parfois de langues et de voix individuelles, diversité littérairement organisée. (DDR, p. 88).

Rappelons également que le terme russe raznorechivost' est également traduit en français par plurilinguisme :

(11) Prozaik-xudoznik vozvodit etu sotzial'nuju raznorechivost' vokryg predmeta do zavereszennogo obraza (DDR, p.92),

L'artiste prosateur érige ce plurilinguisme social à l'entour de l'objet jusqu’à l'image parachevée. (DDR, p. 102)

De même, le terme russe raznorechie, possédant la même racine que le nom raznorechivost' et l'adjectif raznorechivyj, est traduit en français, entre autres, par polylinguisme :

(6) Raznorechie, vvodimoe v roman,- eto chuzaja rech' na chuzom jazykie, sluzaszczaja prelomlennomu vyrazeniju avtorskix intentzij (DDR, p.137).

Le polylinguisme introduit dans le roman, c'est le discours d'autrui dans le langage d'autrui. (DDR, p.145).

On constate que les mêmes termes russes raznogolosnitza, raznorechivost' et raznorechie sont tantôt traduits en français par des équivalents, comme plurivocalité, multiplicité des voix ou (dans d'autres occurrences) polyphonie, qui font apparaître la dimension vocale, sans que cela soit forcément motivé ; tantôt par les notions un peu floues de plurilinguisme et de polylinguisme qui font disparaître la dimension vocale, et dont l'adéquation paraît tout aussi problématique.

De façon générale, les traductions françaises font référence, aussi vague soit-elle, à une pluralité discursive, énonciative et phonique, en somme à une hétérogénéité énonciative, sans toutefois faire apparaître l'aspect de différence voire de divergence discursive qu'introduit razno ('de manière différente, différemment'), dans la série des termes russes. En particulier, le terme raznorechie, n'appartenant pas à la langue usuelle, semble signifier quelque chose comme "hétérogénéité discursive impliquant une confrontation des points de vue divergents » (Nowakowska 2005: 22). Ainsi la traduction de ce terme par plurivocalité semble ne pas être exacte.

Parallèlement au terme raznorechie, la traduction du mot raznoglosnitza, en particulier dans DDR, retient l'attention. Le terme raznogolosnitza (composé du préfixe razno- et de la racine golos) signifie habituellement en russe : dissonance, discordance, divergence d'opinions. La voix (golos dans la racine de ce mot) est, dans ce cas, dématérialisée et assimilée à l'opinion, au point de vue. Or, cette traduction est très rare dans la version française (seulement deux occurrences, p. 90 et 107). Toutes les autres occurrences (dix au total) du terme raznoglosnitza sont traduites par plurivocité. Le terme russe mnogogolosie, composé du préfixe mnogo- (plusieurs, beaucoup) et golos (voix), qui peut signifier également polyphonie, correspondrait plus exactement à plurivocalité, plurivocité ou diversité des voix. Ainsi, on a l'impression que le sens premier du mot raznoglosnitza (divergence d'opinions, dissonance) se trouve dilué dans les termes, somme toute, généraux de plurivocité, plurivocalité ou diversité de voix qui font certes référence à la voix (golos), à la vocalité contenue dans la morphologie du substantif russe raznogolosnitza, mais ne font pas apparaître la nuance de discordance, de divergence qui devrait conduire en français à en donner une lecture en termes de divergence de points de vue, d'opinions. On notera une certaine 
contradiction entre la traduction française par plurivocalité, où la dimension vocale semble faire référence à la voix dans sa matérialité, et le terme russe raznogolosnitza, qui fait entendre certes le mot golos (voix), mais employé dans l'acception dématérialisée d'opinion, discours. Cette lecture résulte de l'emploi polysémique de golos, qui réfère, parfois simultanément, aux dimensions corporelle, discursive et narratologique.

On peut dire que globalement les traductions françaises font apparaître la dimension vocale, là où, dans le texte original, elle n'était pas vraiment explicite. Ce qui est peut-être à l'origine de l'importance qu'a prise cette dimension dans la réception française de Bakhtine, et dans les travaux qui se situent dans le prolongement de sa problématique.

\section{Voix et point de vue dans l'approche polyphonique}

La question de l'hétérogénéité énonciative a suscité, dans les travaux français, deux types de lecture des écrits de Bakhtine : (i) dans le cadre de l'analyse du discours française, à partir du terme de dialogisme ; (ii) dans le cadre pragmatico-énonciatif d'O. Ducrot et de ses disciples, à partir du terme de polyphonie (Bres et Rosier 2008). Les deux font travailler le terme de voix en emprunt à Bakhtine, mais dans des directions différentes.

On suivra dans un premier temps le traitement qui est accordé à voix, rapidement doublé par point de vue dans l'approche polyphonique, d'abord chez O. Ducrot, ensuite chez deux de ses continuateurs : H. Nølke, L. Perrin.

\subsection{O. Ducrot : de la voix au point de vue, ou d'un tâtonnement, l’autre...}

Remarquons pour commencer qu'O. Ducrot, lorsqu'il introduit le terme de polyphonie pour la première fois dans son travail, en 1981, parle de voix. Après avoir distingué locuteur et énonciateur, il ajoute :

(12) ma thèse permet, lorsqu’on interprète un énoncé, d'y entendre s'exprimer une pluralité de voix, différentes de celle du locuteur, ou encore comme disent certains grammairiens à propos des mots que le locuteur ne prend pas à son compte, mais met, explicitement ou non, entre guillemets, une « polyphonie. (1981: 44)

Et effectivement, le terme d'origine grecque polyphonie peut se traduire littéralement par 'pluralité de voix'.

On s'attachera ensuite à la façon dont, dans le texte fondateur de 1984, voix est progressivement remplacée par point de vue. O. Ducrot commence par avancer que " pour Bakhtine, il y a toute une catégorie de textes, et notamment de textes littéraires, pour lesquels il faut reconnaître que plusieurs voix parlent simultanément, sans que l'une d'entre elles soit prépondérante et juge les autres » (p. 171). Le terme de voix, dans cette première occurrence, se voit employé en usage et en mention (cf. " pour Bakhtine »). L'auteur le fait sien ensuite sans réticence aucune, comme p. ex. lorsqu’il déclare que «c'est l'objet propre d'une conception polyphonique du sens que de montrer comment l'énoncé signale, dans son énonciation, la superposition de plusieurs voix » (op. cit. : 183). Et, dans la première partie de cette étude, il parlera tantôt de voix tantôt de point de vue, sans définir ces termes et apparemment en équivalence synonymique. Cependant dès lors qu’il est conduit à poser, à côté du locuteur, responsable de l'énoncé, une autre forme de subjectivité qu'il nomme énonciateur, ainsi définie :

(13) J’appelle « énonciateurs » ces êtres qui sont censés s'exprimer à travers l'énonciation, sans que pour autant on leur attribue de mots précis ; s’ils «parlent », c'est seulement en ce sens que l'énonciation est vue comme exprimant leur point de vue, leur position, leur attitude, mais non pas, au sens matériel du terme, leurs paroles. (p. 204) 
voix va tendre à s'effacer au bénéfice de point de vue. Ce qui est parfaitement cohérent : à partir du moment où les énonciateurs sont définis comme des instances sans parole remarquons les guillemets qui modalisent parler -, le terme de voix, même métaphorique, n'est plus pertinent, car trop lié à la parole dans sa matérialité. Point de vue, qui fait appel à un autre sens, celui de la vue, déplaçant la métaphore, permet par contre de nommer cette altérité sans passer par la matérialité des mots. Pour autant, remarquons l'énumération : « leur point de vue, leur position, leur attitude » : point de vue est glosé, remplacé par position, attitude, et non pas défini comme une notion centrale. Le terme semble être employé par défaut d'un terme plus approprié, pour ce qu'il permet d'éviter : que l'énonciateur ne soit lié à des mots précis. Rappelons l'exemple qui accompagne cette définition :

(14) Et ce même Néron, que la vertu conduit, / Fait enlever Junie au milieu de la nuit. (Agrippine, in Britannicus, Racine)

qui se voit commenté ainsi : "La relative est destinée à exprimer non pas le point de vue d'Agrippine mais celui d'Albine (op. cit. : 204) ». Selon O. Ducrot, l'énoncé La vertu conduit (Néron) ne rapporte pas les mots d'Albine, on ne saurait entendre là à proprement parler sa voix; en revanche on peut facilement le mettre en relation avec la façon dont elle voit Néron...

Et pour mieux définir ce qu'il entend par énonciateur et donc point de vue, O. Ducrot développe ensuite deux comparaisons : avec le théâtre, d'abord, puis avec le roman, plus précisément avec les propositions narratologiques de Genette. C'est celle-ci qui retiendra notre attention. L'auteur brosse une mise en relation que l'on peut synthétiser par le petit tableau suivant :

\begin{tabular}{|c|c|c|}
\hline $\begin{array}{l}\text { narratologie } \\
\text { polyphonie }\end{array}$ & $\begin{array}{l}\text { narrateur } \\
\text { locuteur }\end{array}$ & $\begin{array}{l}\text { centre de perspective, point de vue } \\
\text { énonciateur }\end{array}$ \\
\hline
\end{tabular}

Il précise notamment :

(15) Le locuteur parle au sens où le narrateur raconte, c'est-à-dire qu'il est donné comme la source d'un discours. Mais les attitudes exprimées dans ce discours peuvent être attribuées à des énonciateurs dont il se distancie - comme les points de vue manifestés dans le récit peuvent être ceux de sujets de conscience étrangers au narrateur. (op. cit., p. 208)

Si l'on se fie à cette citation, le terme de point de vue semble emprunté à la narratologie, en tout cas il se voit légitimé en tant que notion issue de cette théorie, comme équivalent du bien peu scientifique « attitude ». Dans la suite de l'article, il ne sera plus guère parlé de voix mais de point de vue, notion qui se voit définie plus précisément p. 218, lorsque traitant de la négation, il est précisé :

(16) L'élément positif que je déclare sous-jacent à l'énoncé négatif n'est pas un énoncé (c'est-à-dire une suite de mots, imputables à un locuteur), mais une attitude, une position prise par un énonciateur vis-à-vis d'un certain contenu, c'est-à-dire d'une entité sémantique abstraite. Quand je parle d'une proposition sous-jacente à « Pierre n’a pas fait grand chose », il ne s'agit pas d'une proposition grammaticale, mais d'une proposition au sens logique, c’est-à-dire d'un objet de pensée.

On remarque cependant qu'en dépit de ce remplacement théorique de voix par point de vue, O. Ducrot, dans l'étude de certains exemples, à plusieurs reprises, use non du vocabulaire de la vue, mais de celui de la parole. Analysant un mot d'esprit (p. 212), il commente : «le client, pris comme le locuteur L, fait exprimer par un énonciateur, assimilé au patron, l’opinion sur le passé du teckel ». Traitant de l’interrogation, il précise (p. 227) : «ces 
énoncés doivent faire apparaître un énonciateur exprimant son doute en ce qui concerne la proposition sur laquelle porte l'interrogation ». Mais qu'est-ce qu' « exprimer une opinion » ou « un doute », si ce n'est du discours ? Comme si la thématique de la voix, chassée par la porte de la théorie, revenait par les fenêtres de l'analyse des exemples...

L'abandon de voix au profit de point de vue est confirmé dans Ducrot 2001. Nous n'avons relevé qu'une seule occurrence de voix, et qui semble comme avoir échappé à la sagacité du scripteur: à la fin de l'article, résumant le projet de la ScaPoLine des polyphonistes scandinaves, il est dit : "son problème est d'intégrer à l'intérieur du texte, pris dans sa totalité, les différentes voix que l'on a reconnues à l'intérieur des énoncés » (2001: 40). Partout ailleurs, il est question de point de vue. Plus même, la dimension scopique impliquée par ce terme fait l'objet d'un filage métaphorique qui la radicalise :

(17) à chaque point de vue, je relie un énonciateur, présenté comme la source de ce point de vue, ou, en filant la métaphore, comme l’œil qui voit. (2001 : 20)

Pour conclure, on dira que Ducrot part en 1980 du terme de voix qui lui est fourni par la vulgate bakhtinienne ; mais, dans le cours même de l'article de 1984, il remplace ce terme, trop incarné, qui ne correspond plus à la définition de l'énonciateur comme instance sans parole, par celui de point de vue, en appui sur la théorisation narratologique de la focalisation. Cette substitution se verra confirmée par la suite, même si Ducrot (i) a quelque difficulté dans ses analyses concrètes à s'en tenir au champ lexical de la vue et à ne pas faire appel au champ lexical de la parole ; et (ii) ne fait pas vraiment accéder le terme de point de vue au statut de notion, dans la mesure où il le double souvent par celui d’attitude, bien peu précis.

\subsection{La ScaPoLine... ou le sacre du point de vue}

Le groupe de recherche scandinave, réuni autour de H. Nølke, confirme le remplacement de voix par point de vue :

(18) la polyphonie, c'est bien évidemment cette présence de différents points de vue, ou de "voix" dans un seul énoncé. (Nølke 1994 : 146)

Notons la modalisation autonymique des guillemets sur le mot voix, qui est donc en usage et en mention: façon de signaler l'inscription en autre de ce terme ou / et son caractère approximatif $^{7}$, alors que point de vue est posé en seul usage, comme le terme sélectionné pour son adéquation. Par la suite, ce terme sera travaillé et défini très précisément. En reprise à O. Ducrot du syntagme « entité sémantique abstraite » (supra (16)), il est posé que :

(19) les points de vue sont des entités sémantiques composées d'une source, d'un jugement et d'un contenu propositionnel. [...] il y a toujours au moins un pdv qui est marqué dans la signification d'une phrase. (2004 : 31)

Ce faisant, la ScaPoLine s'éloigne doublement d'O. Ducrot: elle introduit une structure interne du "pdv ${ }^{8}$ (Nølke 2006 : 249), qui dès lors n’est plus un terme approximatif pour évoquer, au niveau linguistique, cette forme de subjectivité que sont les énonciateurs. Mais surtout, en posant que " chaque énoncé contient au moins un pdv simple dont le contenu sémantique est posé » (2004 : 33), Nølke fait du point de vue un élément qui structure le sens de tout énoncé, fût-il énonciativement de la plus grande simplicité comme p. ex. il fait beau. De sorte que la notion de point de vue, qui chez O. Ducrot visait à nommer le rôle d'une

\footnotetext{
${ }^{7}$ On retrouve pareille mise à distance énonciative dans Nølke $2006: 250$.

${ }^{8}$ Notons la siglaison de point de vue en $p d v$, qui signale son statut conceptuel.
} 
instance autre que celle du locuteur, à savoir l'énonciateur, sort de ce rôle ancillaire pour devenir une notion centrale de la théorisation scandinave. Comme telle, elle se complexifie : elle se voit refendue en pdv simple, pdv hiérarchique et pdv relationnel ; et entraîne la disparition de la notion d'énonciateur.

On dira, pour conclure, que la ScaPoLine radicalise le mouvement de substitution de point de vue à voix repéré dans les travaux d'O. Ducrot : voix, lorsque ce terme apparaît, est tenu à distance par les guillemets; point de vue passe du statut de métaphore à celui de concept rigoureusement défini.

\subsection{Perrin : la voix du locuteur, le point de vue des énonciateurs}

Dans différents travaux, L. Perrin, retenant les deux termes de voix et de point de vue, en propose une articulation très précise, qui lie le premier au seul locuteur et le second à l'énonciateur. En relecture de Ducrot (1984), il distingue clairement ce qui était quelque peu tâtonnant en opposant deux types de polyphonies :

- le premier consiste en «l'intégration de voix étrangères associées à d'autres locuteurs » (2006 : 22). Il concerne la forme de l'expression, et se réalise par le discours direct et la modalisation autonymique. Par le discours rapporté direct, le locuteur citant fait entendre les mots même d'un autre locuteur, ceux de la voix citée qui les prend en charge ;

- le second, plus abstrait, consiste en «l'intégration de différents points de vue » (ibid.) émanant de différents énonciateurs. Il concerne le contenu de l'expression, et se réalise par le discours indirect, toutes les formes de reformulation, la négation, etc. Dans ce cas, le locuteur citant est responsable des mots utilisés mais pas du point de vue qui s’y exprime, qui est celui d'un autre énonciateur. Soit donc :

$\begin{array}{lll}\text { voix } & \text { locuteur } & \text { forme de l'expression } \\ \text { point de vue } & \text { énonciateur } & \text { contenu de l'expression }\end{array}$

Dans le second cas, il y a « une sorte de distorsion, de désolidarisation entre énonciation et point de vue, entre prise en charge de la forme linguistique et du contenu d'un discours. » (op. cit. : 26), ce que nous illustrerons par un ex. emprunté à l'auteur :

(20) Marc Dutroux le gentil, le sauveur, le philanthrope, le philosophe, le scrupuleux, la victime, le repentant. Tel est le portait ahurissant que l'accusé le plus honni de Belgique a dressé de lui-même durant son premier interrogatoire. (Le temps, 4 / 3 / 04)

L. Perrin commente ainsi : « Le locuteur assume le choix des mots et des formulations [...] mais pas le point de vue que ces mots expriment qui n'est que l'objet de référence de ce qu'il cherche à communiquer » (p. 25). Effectivement, il semble bien que les différents $\mathrm{SN}$ : le gentil, le sauveur, etc. soient les mots du locuteur (ici le scripteur journaliste) et non ceux de l'énonciateur (Marc Dutroux), mais que ces mots expriment le point de vue de l'énonciateur sur lui-même, non celui du locuteur.

La distinction très fine que fait L. Perrin de la voix et du point de vue lui permet de saisir et de décrire des faits discursifs très pointus ${ }^{9}$. Elle a d'autre part le mérite de s’appuyer sur la vieille distinction discours direct / discours indirect, qu'elle permet de revisiter et de complexifier.

\subsection{Point de vue : romances sans paroles?}

Essayons d'évaluer la pertinence de la notion de point de vue, globalement, dans l'approche de l’hétérogénéité énonciative initiée par O. Ducrot : elle permet d'appréhender une forme de

\footnotetext{
${ }^{9}$ Cf. ici même son article.
} 
subjectivité infra-verbale, en remplacement du terme par trop incarné de voix (ou en limitation du champ d'action de ce terme chez L. Perrin). Elle est en accord avec notre intuition des faits langagiers: il semble bien que la subordonnée relative en (14) "(Néron) que la vertu conduit» ne rapporte pas les mots d'Albine; pas plus que les SN « (Marc Dutroux) le gentil, le sauveur, le philanthrope (...) » ne rapportent les propos de l'accusé sur lui-même en (20). Et il est tout aussi évident que ces éléments sont de quelque façon liés à Albine et à Marc Dutroux, qu'ils sont bien là le signe de leur subjectivité : une subjectivité qui procède non de l'énonciation elle-même de ces mots mais de quelque chose de plus abstrait saisi par la métaphore du point de vue, le regard ${ }^{10}$ d'un énonciateur, que traduiraient ces mots : regard d'Albine sur Néron, regard de Dutroux sur lui-même.

Cette conclusion, à laquelle conduisent les travaux que nous avons présentés, nous ne la partageons pas, pour les deux raisons suivantes :

(i) O. Ducrot, comme nous l'avons dit, pour mettre en place la distinction locuteur / énonciateur, s'appuie sur l'analyse narratologique proposée par Genette. On peut se demander si en empruntant le terme de point de vue, qui en narratologie relève de la question Qui voit ?, et en le faisant servir à des questions d'énonciation, $O$. Ducrot n’opère pas un détournement théorique dans la mesure où Genette pourfend la " confusion » entre perspective qui relève du mode (qui voit ?) et voix (qui parle ?) (1972 : 203). On peut lever cette possible critique en avançant que précisément, dans les cas de polyphonie comme (14) et (20), il ne s'agit pas de la parole des énonciateurs, de leurs mots, mais de leur point de vue, c'est-à-dire de quelque chose de plus abstrait, d'antérieur à la verbalisation, mais de fortement lié à la subjectivité de l'énonciateur, que les mots utilisés auraient seulement pour fonction de traduire. Certes. On fera alors remarquer que le terme de polyphonie - littéralement 'plusieurs voix'-, dont O. Ducrot soulignait la pertinence lorsqu'il appréhendait le phénomène comme celui de la « pluralité des voix » dans un même énoncé (supra (12)), devient, s’il s’agit de la pluralité des points de vue quelque peu disconvenant, et que polyscopie serait plus pertinent ${ }^{11}$. Mais ce n'est là que broutille. Le fond de notre opposition à point de vue est ailleurs, et autrement sérieux.

(ii) Pour le dire tout de go, il nous semble que la position théorique qui sous-tend l'approche de l'hétérogénéité énonciative en termes de point de vue est idéaliste, à savoir qu'elle s'inscrit dans un paradigme linguistique qui considère que les mots ne font qu'actualiser un contenu préalablement pensé (cf. dans la citation d'O. Ducrot supra (16), les termes d' " entité sémantique abstraite ", de " proposition au sens logique, c'est-à-dire d'un objet de pensée »), qui serait donc à la fois antérieur et extérieur aux mots pour le dire. On lui opposera une approche matérialiste, plus précisément celle de l'analyse du discours qui pose que le sens vient aux mots moins d'avoir été préalablement pensé par le sujet parlant que déjà énoncé dans des discours antérieurs ; que donc l'interdiscours précède le discours qui toujours en procède, qu'il n'y a d'extérieur au discours - et il nous semble que c'est cette position d'extériorité qu'occupe la notion de point de vue dans l'approche polyphonique - que pour le sujet parlant qui s'illusionne... Qu'est-ce que ce choix induit concrètement dans l'analyse des fragments considérés précédemment à partir de la notion de point de vue ? Reprenons chacun d'eux :

(14) Et ce même Néron, que la vertu conduit, / Fait enlever Junie au milieu de la nuit. (v. 53-54)

Nous l'avons vu : O. Ducrot analyse la subordonnée relative comme exprimant le point de vue de l'énonciateur Albine, sans rapporter ses mots. Remarquons tout d'abord que cette

\footnotetext{
${ }^{10}$ Notons que Nølke intitule significativement deux de ses ouvrages Le regard du locuteur (1993, 2001, Kimé).

${ }^{11}$ Polyscopie est avancé par M. Wilmet, pour distinguer la polyphonie ou « concert de voix » de la diversité des points de vue (1997/2003 : 481).
} 
analyse n'est pas tout à fait exacte : si nous relisons cette scène de Britannicus, il apparaît que, dans sa défense de Néron, Albine use elle-même, quelques vers plus haut, du mot de vertu :

(21) (...) Enfin Néron naissant / A toutes les vertus d'Auguste vieillissant. (v. 29-30)

De sorte qu'Agrippine reprend bien un mot du discours d'Albine, "vertu ", qui fait donc l'objet, implicitement, d'une modalisation autonymique : il est en usage et en mention ${ }^{12}$. Mais ceci n'est pas déterminant pour notre propos. On analysera le discours d'Agrippine non seulement comme réponse dialogale à celui d'Albine mais aussi (i) comme dialogiquement orienté vers lui, ce que signale entre autres la reprise du mot vertu, à savoir que le discours d’Agrippine trouve sa source dans celui de Junie ; et, (ii) complémentairement, la relative, comme discours que le locuteur-énonciateur $\mathrm{E}_{1}$ (correspondant à Agrippine) impute non sans ironie à l'énonciateur $\mathrm{e}_{1}{ }^{13}$ (correspondant à Albine).

Ce que nous analysons comme dimension discursive du dialogisme est tout aussi évident dans l'ex. (20) :

(20) Marc Dutroux le gentil, le sauveur, le philanthrope, le philosophe, le scrupuleux, la victime, le repentant. Tel est le portait ahurissant que l'accusé le plus honni de Belgique a dressé de lui-même durant son premier interrogatoire. (Le temps, 4 / 3 / 04)

Notons que les syntagmes nominaux apposés «le gentil, le sauveur », etc. sont présentés comme rapportant fidèlement (tel) un discours tenu par Marc Dutroux (portrait, dressé). Comme dans l'exemple précédent, le dialogisme de cet énoncé tient d'abord à son dialogue avec un autre discours (celui de Dutroux, présupposé par le terme portrait). Mais nul n’est dupe : ces mots ne sont certainement pas verbatim ceux de Marc Dutroux, mais ceux que le locuteur prête à l'énonciateur $\mathrm{e}_{1}$, mots qu'il imagine non sans exagération qu'il pourrait énoncer. De sorte que plutôt que d'y voir les mots du locuteur énonçant le point de vue de l'énonciateur, on analysera cet énoncé comme typiquement bivocal pour reprendre un terme bakhtinien, c'est-à-dire que s'y font entendre deux voix, celle de l'énonciateur citant et celle de l'énonciateur cité. Nous venons de réintroduire le terme de voix : nous allons voir qu'il permet de décrire les phénomènes d'hétérogénéité énonciative en évitant le recours à la notion idéaliste de point de vue. Mais dès à présent, soulignons ce qui nous apparaît comme une différence, et peut-être un gain: l'approche polyphoniste pose une distorsion entre " énonciation et point de vue, entre prise en charge de la forme linguistique et (prise en charge) du contenu » (Perrin, supra). Notre approche du dialogisme analysera ce type d'énoncé non comme dissociation de deux instances mais au contraire comme leur interaction vive : l'énonciateur principal $\mathrm{E}_{1}$ prête à l'énonciateur enchâssé $\mathrm{e}_{1}$ un discours qui pourrait être le sien, tout en en profitant au passage pour le déformer, le caricaturer.

Nous avons vu que Ducrot 1984 abandonnait le terme de voix pour celui de point de vue afin de traiter des énoncés dans lesquels n'apparaît pas la parole rapportée dans sa matérialité (ses « mots »). Il nous semble qu'il est conduit logiquement à cette substitution parce qu'il ne dispose pas de la catégorie de discours ${ }^{14}$, entendu non seulement comme ce qui est effectivement dit, mais aussi comme ce qui a pu être dit et ce qui pourra être dit ; c'est-à-dire

\footnotetext{
${ }^{12}$ Certes avec un jeu sur le nombre (du pluriel au singulier), et l'effacement du complément de nom ( « d'Auguste vieillissant »), qui permet l'actualisation de sens légèrement différents, ce qui actualise la figure du polyptote.

${ }^{13}$ Pour la façon dont nous utilisons les notions de locuteur et d'énonciateur, cf. infra 3.

14 Nous semble significative cette remarque de Ducrot à propos de la façon dont il adapte une recherche de Sperber et Wilson sur l'ironie: « J'ai substitué l'expression «faire entendre une voix » à leur expression originale qui est « mentionner un discours » $(1984: 210)$.
} 
dont la matérialité n'est pas liée strictement à des mots précis mais consiste en un ensemble de formulations en relation paraphrastique.

Pour ces deux raisons, nous choisissons dans nos propres travaux, pour "pêcher " l'hétérogénéité énonciative, d'user non de la notion de point de vue, intuitivement " parlante » et d'une opérativité certaine, mais liée à une conception non discursive de la production de sens que nous ne partageons pas ; mais de celle de voix, qui alors même qu'elle peut paraître comme procédant d'une métaphore abusive, se révèle pleinement pertinente pour une approche qui se situe dans les cadres de l'analyse du discours.

\section{Dialogisme, discours et voix}

Les recherches qui, dans les cadres de l'analyse du discours, rencontrent la question de l'hétérogénéité énonciative, - nous pensons notamment à Authier-Revuz (1995) et à Moirand (2007) - le font à partir du terme de dialogisme et usent tendanciellement du seul terme de voix ${ }^{15}$ de façon spontanée, sans chercher à lui donner un statut terminologique. Le travail notionnel qui s'est fait dans les cadres de l'approche polyphonique et que nous avons présenté supra reste à faire pour l'approche dialogique. Ce que nous entreprenons ici même, par une réflexion sur nos propres études en la matière.

Nous le reconnaissons bien volontiers : jusqu'à ce jour, nous avons usé du terme de voix comme s'il allait de soi... non sans jouer à le carnavaliser parfois, comme dans ce titre d'article qui convoquait allusivement Jeanne d'Arc : "Entendre des voix » (Bres 1998)... Passons donc aux choses sérieuses, et rappelons brièvement pour commencer notre façon d'analyser l'énoncé dialogique (Bres et Verine 2003, Bres et Nowakowska 2006), à partir d'une occurrence d'interaction verbale retenue pour sa simplicité :

(22) Petit rituel quotidien du thé dans le laboratoire, vers $16 \mathrm{~h} 30$ : le directeur fait chauffer de l'eau dans la bouilloire et lance, à la cantonade dans le couloir, invariablement, l'énoncé suivant :

- $\quad$ y a de l'eau chaude

dans une phonologie à fort substrat occitan, qui s'éloigne de la prononciation française notamment par la prononciation du e dit sourd [ø], et par l'ouverture du o fermé [o] de chaude.

Nous distinguons, dans cet énoncé [E], l'instance du locuteur $\left(\mathrm{L}_{1}\right)$, qui actualise phonétiquement l'énoncé dans sa dimension locutoire de dire, de l'instance de l'énonciateur $\left(E_{1}\right)$ qui l'actualise aux différents niveaux déictique, syntaxique, praxémique et modal. Locuteur et énonciateur sont dans cet énoncé co-référentiels, et correspondent contextuellement au directeur.

(23) Un jour que le directeur a laissé passé l’heure, une doctorante le remplace dans cette tâche et lance de semblable façon à la cantonade :

- y a de l'eau chaude

en contrefaisant la prononciation francitane du directeur (notamment par une plus grande ouverture du [o] de chaude), ce qui provoque le rire général.

\footnotetext{
${ }^{15}$ A l'exception de P. Siblot (2001) qui met en relation point de vue inscrit dans la nomination et dialogisme. Mais ledit point de vue ne correspond pas à une forme de subjectivité pré-verbale: il est inscrit dans la nomination par les praxis et par les discours qui procèdent de ces praxis et qui les accompagnent.
} 
Les interactants ont perçu dans l'énoncé de la doctorante l'écho de l'énoncé du directeur, ainsi que sa mimesis ironique. Ce second énoncé est dialogique en ce qu’il est hétérogène énonciativement. On distingue, à des fins d'analyse, deux actes d'énonciation :

- l'énoncé enchâssant [E] [y a de l'eau chaude], avec son locuteur $\mathrm{L}_{1}$ et son énonciateur $E_{1}$, qui correspondent contextuellement à la doctorante ;

- l'énoncé enchâssé [e], qui, dans la mesure où [E] en est une exacte reprise-écho, a la même forme que lui, à savoir [y a de l'eau chaude], mais dispose d'instances différentes, notées $l_{1}$ pour le locuteur et $\mathrm{e}_{1}$ pour l'énonciateur, qui correspondent contextuellement au directeur.

Ces deux actes d'énonciation en interaction dans l'énoncé dialogique sont dans une relation non pas d'égalité et de (relative) indépendance, comme le sont p. ex. ceux de deux tours de parole dans un texte dialogal, mais de hiérarchie et de dépendance, ce que signale le jeu typographique: les majuscules, pour les paramètres de la structure enchâssante; les minuscules, pour ceux de la structure enchâssée. On note la hiérarchisation énonciative des deux énoncés de la sorte: $(\mathrm{E}(\mathrm{e}))$. De quelle pertinence peut être la notion de voix ? On s'attachera à mettre successivement en relation ladite notion avec l'instance des locuteurs, puis avec celle des énonciateurs.

\subsection{Voix et locuteur(s)}

Le terme de voix, au niveau de la dimension locutoire de l'actualisation, n'a rien de métaphorique dans une occurrence orale comme (23): si l'on parle de la voix de $\mathrm{L}_{1}$ (correspondant à la doctorante), il s'agit bien de la voix dans toutes ses dimensions telles que les pose la phonétique (hauteur, intensité, durée, timbre), à savoir comme une signature sonore, qui fait que les interlocuteurs l'identifient, dans son irréductible individualité, comme étant celle de la doctorante B. L. Mais qu'en est-il de la voix de $\mathrm{l}_{1}$ (locuteur cité) ? Notons tout d'abord qu'à la différence de celle de $\mathrm{L}_{1}$, elle n'est pas directement présente, mais seulement rapportée, et c'est de la plus ou moins bonne qualité de cet acte de mimésis qu'elle sera reconnue comme faisant référence à $l_{1}$, ici le directeur. Dans le cas précis, la mimésis porte sur un élément phonique ethniquement identificateur: la réalisation de certaines voyelles. Moins fréquemment, la mimésis peut porter sur une particularité de la locution de $l_{1}$, le bégaiement ou le zézaiement p. ex.. Le dialogisme, au niveau locutoire, tient à l'interaction de la voix réelle de $L_{1}$ avec certaines caractéristiques de la voix représentée de $l_{1}$, interaction qui se manifeste sous la forme d'une hétérogénéité phonique. Sans dresser les différentes formes de cette interaction, notons trois cas :

- La mimesis de la voix de $l_{1}$ par celle de $L_{1}$ peut être (presque) parfaite : l'interlocuteur ne percevra pas le dialogisme de l'énoncé et croira avoir effectivement affaire à la voix de 11 . Le gag téléphonique joue sur cette confusion : p. ex. en septembre 2005, un comique imitant parfaitement la voix du président Chirac avait réussi à se faire passer pour lui en contactant le capitaine de l'équipe de France de football, à qui il avait demandé que les joueurs français, en signe de participation à une bonne cause, mettent la main sur leur cœur pendant la Marseillaise, ce qu'ils avaient fait en toute bonne foi. Côté émission cependant, l'énoncé relevait du dialogisme : c'était bien la voix de l'imitateur $\left(\mathrm{L}_{1}\right)$ qui contrefaisait celle de J. Chirac $\left(l_{1}\right)$.

- L’énoncé dialogique apparaît le plus souvent sans marquage dialogique de la voix de $l_{1}$ : à l'oral, le discours direct ou le discours direct libre p. ex., qui se présentent comme rapportant les propos de l'énonciateur $\mathrm{e}_{1}$, ne miment que rarement la voix du locuteur enchâssé ${ }^{16}$ :

\footnotetext{
${ }^{16}$ Cf. ici même l'article de B. Verine.
} 
(24) (conversation. Une femme parle des déplacements en train d'une amie)

Mathilde quand elle va chez son fils à Clermont elle passe par Alés / pas par Nimes non avec mes valises je suis chargée et j'ai à peine le temps de changer de train alors que par Alés elle a vingt minutes de battement

La locutrice $\mathrm{L}_{1}$ cite les paroles de son amie Mathilde sans aucun changement de voix : c'est seul le contexte qui, en l'absence de toute mimésis de la voix de $l_{1}$, permet de comprendre que les déictiques personnels et temporels de l'énoncé en italiques réfèrent à un autre énonciateur $\mathrm{e}_{1}$ (l'amie Mathilde), différent de $\mathrm{E}_{1}$.

- Nous avons dit que, dans l'énoncé dialogique, la voix de $l_{1}$ pouvait être au mieux mimée par celle de $\mathrm{L}_{1}$, mais qu'elle n’apparaissait pas elle-même dans sa matérialité. Ajoutons que la technique moderne de l'enregistrement permet de lever cette impossibilité. Soit p. ex. dans les informations à la radio :

(25) les militants socialistes semblent fatigués de cette course suicidaire à la présidence qui hypothèque le futur

C’est la voix pré-enregistrée de $l_{1}$ (militant socialiste) qui verbalise le syntagme « course suicidaire à la présidence ", à l'intérieur de l'énoncé verbalisé par la voix de $\mathrm{L}_{1}$ (journaliste).

Jusqu'à présent, nous avons parlé de la pertinence de la notion de voix pour l'instance des locuteurs dans le discours oral. Qu'en est-il à l'écrit ? Le correspondant de la voix dans le code écrit nous semble être l'écriture, qui, comme celle-ci, identifie l'individu dans son unicité ; et fonctionne comme signature graphique : les «corbeaux » le savent bien qui au lieu d'écrire leurs lettres de menace ou de dénonciation à la main, découpent des caractères imprimés pour garder leur anonymat... L'ère de la machine à écrire, et plus encore de l'ordinateur, réduit à peau de chagrin l'écriture, et pour ce qui nous concerne sa possible dimension dialogique. Pour autant, on distinguera au moins deux cas d'utilisation dialogique de l'écriture :

- le scripteur $\mathrm{S}_{1}{ }^{17}$ mime orthographiquement certains traits de la phonie de $\mathrm{l}_{1}$. Ce peut être la réalisation de certains phonèmes, comme dans l'occurrence suivante :

(26) la narratrice raconte, par courriel, à une amie le petit scandale provoqué par une commune amie slave, dans un magasin de chaussures :

- j’ai entendu sa voix en colère qui criait : «Alorrrs elle arrrrive cette chaussurrrrre oui ou merrrrde !!! », je me suis précipitée (...)

La multiplication du graphème $r$ est censée faire entendre la voix de $l_{1}$, par le biais d'un trait de sa phonie : la prononciation roulée de la liquide [r].

Ce peut être également un trait particulier de la locution de $\mathrm{l}_{1}$, comme le bégaiement du père Grandet signifié par la répétition de la syllabe initiale des mots et la multiplication des graphèmes vocaliques :

(27) - Mon... on...on... sieur le pré... pré... pré... président, vouoouous di... di... disiiieez que la faaaaiiillite...(Eugénie Grandet, Balzac)

- le scripteur $S_{1}$ reproduit certains traits de la graphie de $s_{1}$, typiquement ses fautes d'orthographe :

(28) Titre d'un article du Monde, écrit par un responsable de l'audiovisuel qui venait de se faire démettre de ses fonctions, en 1994, alors qu’Edouard Balladur était premier ministre :

- Édouard m'a tuer

\footnotetext{
${ }^{17}$ On parlera de $\mathrm{S}_{1}$ pour le scripteur de l'acte enchâssant, et de $\mathrm{s}_{1}$ pour celui de l'acte enchâssé.
} 
La faute d'orthographe (tuer à la place de tué) convoque dialogiquement un autre énoncé produit dans le cadre d'un assassinat crapuleux qui avait fait la une de l'actualité : une femme avait été assassinée dans sa cave. Sur la porte barricadée de celle-ci, les enquêteurs avaient trouvé, inscrit avec le sang de la victime, le graffiti Omar m'a tuer... Ladite faute par laquelle $\mathrm{S}_{1}$ (correspondant au responsable de l'audiovisuel) mime l'orthographe de $\mathrm{s}_{1}$ (la femme assassinée (?)) est le marqueur dialogique qui permet de comprendre (28) comme le détournement du graffiti.

On peut dire que, dans les occurrences du type de (26) et de (27), est signalée, dans un énoncé écrit, la voix d'un locuteur enchâssé $l_{1}$; dans les (rares) occurrences du type de (28), l'écriture d'un scripteur enchâssé $\mathrm{s}_{1}$.

Le terme de voix, et ce dans une acception non métaphorique, nous semble donc tout à fait pertinent pour décrire le dialogisme au niveau de l'instance des locuteurs. Qu'en est-il au niveau de celle des énonciateurs?

\subsection{Voix et énonciateurs}

A la différence des locuteurs, les énonciateurs ne sauraient avoir une voix au sens physique du terme dans la mesure où cette instance actualise l'énoncé dans sa dimension non pas locutoire mais énonciative. De sorte que, lorsque dans le Dictionnaire des termes et concepts de la praxématique, nous définissions le dialogisme comme " capacité de l'énoncé à faire entendre, outre la voix de l'énonciateur, une ou plusieurs autres voix qui le feuillettent énonciativement » (Détrie et al., 2001 : 83), nous faisions de ce terme un usage forcément métaphorique, en emprunt aux traductions des textes bakhtiniens.

En relecture des analyses d'O. Ducrot et de L. Perrin, nous avons proposé d'analyser les occurrences (14) et (20) non comme distorsion de deux instances mais comme interaction de deux discours : celui de $e_{1}$ et celui de $E_{1}$, sous la forme d'un énoncé que $E_{1}$ prête ironiquement à $\mathrm{e}_{1}$; et on a proposé de parler de bivocalité, dans la mesure où s'y laisseraient entendre deux voix: celle de $\mathrm{e}_{1}$, et celle de $\mathrm{E}_{1}$. Il nous reste à expliquer le passage du syntagme "deux discours », au syntagme "deux voix ». La métaphore de la voix est-elle pure facilité sans grande pertinence, ou permet-elle au contraire de saisir un fonctionnement du dialogisme, et plus encore un mode de circulation des discours ?

Notons tout d'abord, pour commencer, que le terme de voix, dans le type d'analyse que nous menons, n'est nullement indispensable et qu'on peut parfaitement définir le dialogisme comme orientation du discours, constitutive et au principe de sa production comme de son interprétation, vers d'autres discours (Bres et Nowakowska 2006 : 23), cette orientation se réalisant comme interaction, marquée ou non marquée. Si donc nous pouvons nous passer du terme de voix, quel intérêt y a-t-il à le conserver? Pour répondre à cette question, nous distinguerons deux grands types d'occurrences.

\subsubsection{De la voix ajoutée}

Le 27 février 2008, un journaliste sportif de la télévision rappelle la difficile victoire footballistique du Paris-Saint-Germain contre Auxerre la veille, et enchaîne, sans aucun changement vocal :

(29) Paris inquiété, Paris malmené mais Paris qualifié

On peut y percevoir le détournement allusif du célèbre énoncé de Ch. de Gaulle dans son discours du 25 août 1944 : 
En (29), l'anaphore qui thématise le toponyme Paris, le troisième membre introduit par la conjonction mais, ainsi que les trois participes passés du premier groupe formant assonance en [e] convoquent l'énoncé (30) du Général. Le locuteur $\mathrm{L}_{1}$ ne reproduit rien de la voix de $\mathrm{l}_{1}$, mais le récepteur, s'il reconnaît (30) sous (29), peut l'entendre dans la mesure où il a entendu (30) prononcé par de Gaulle (ou que connaissant la voix de de Gaulle, il l'imagine sur cet énoncé). De sorte que la réception dialogique de (29) peut se faire en ajout de la voix de $l_{1}$.

Dans les occurrences de ce type, où l'énoncé [e] avec lequel interagit [E] est accompagné dans notre mémoire discursive de sa réalisation sonore (ou de la connaissance de la voix de 11), il semble bien qu'on puisse parler de la voix de $l_{1}$ : si acoustiquement elle n'y est pas, l'interprétation dialogique la convoque, la fait entendre.

Mais tel n’est pas le cas le plus fréquent. Bien souvent, la perception de l'énoncé [e] dans [E] ne déclenche pas dans notre mémoire de souvenir de la voix de $l 1 l_{1}$, et plus encore $l_{1}$ n'a pas à proprement parler de voix.

\subsubsection{Discours cherche voix...}

Reprenons l'ex. (20) :

(20) Marc Dutroux le gentil, le sauveur, le philanthrope, le philosophe, le scrupuleux, la victime, le repentant.

Nous avons analysé les syntagmes en apposition comme interaction du discours de $\mathrm{E}_{1}$ (correspondant au scripteur) avec le discours potentiel de $\mathrm{e}_{1}$ (correspondant à Dutroux). Pouvons-nous dire que dans ces syntagmes, nous entendons la voix de Dutroux ? Dans les faits, non: à la différence de l'exemple précédent, nous n'avons jamais entendu parler Dutroux, nous ne saurions donc rajouter sa voix. Dire que cet énoncé est bivocal est donc quelque peu un abus de langage. Et pourtant... cette facilité nous paraît faire sens : si on a tendance à parler de voix autre quand on repère une altérité énonciative, n’est-ce pas parce que, dans les interactions verbales dialogales, nous percevons l'autre dans son altérité d'abord par la voix, et que dans les situations d'interaction in absentia, p. ex. téléphoniques, c'est la voix en tant que signature sonore qui permet l'identification du locuteur ? Ce que signalent de belle manière ces dernières lignes de L'Amant de Duras :

(31) Des années après la guerre, [...] il était venu à Paris avec sa femme. Il lui avait téléphoné. C’est moi. Elle l'avait reconnu dès la voix. (...). (Duras, L'Amant)

Parler de voix, métaphoriquement, pour des ex. comme (20), nous semble s'inscrire dans ce fond d'expérience commune selon lequel le discours, aussi écrit soit-il, aussi impersonnel ou « transpersonnel » soit-il - le discours de la sagesse, de la doxa, de l'opposition... - nous paraît non seulement énoncé, mais incorporé. De sorte que sa perception, et plus encore son identification, convoque une image sonore - fût-elle totalement imaginaire -, ce que capte le terme de voix : la voix de la sagesse, de la doxa, de l'opposition, du peuple... Notre propos

\footnotetext{
${ }^{18}$ Il semble d'ailleurs que le discours footballistique soit coutumier du détournement dialogique de cet énoncé. Une brève recherche sur Internet permet de relever, sur le site Omarseillais.com le titre suivant : "Paris SG : Paris blessé mais Paris libéré » (16. 10. 2005)...
} 
nous semble rejoindre ce que dit M. Foucault de l'énoncé, ou H. Meschonnic du discours, dans d'autres cadres conceptuels :

(32) Pourrait-on parler d'énoncé si une voix ne l'avait pas articulé, si une surface n’en portait pas les signes, s'il n’avait pris corps dans un élément sensible et s'il n’avait laissé trace - ne serait-ce que quelques instants - dans une mémoire ou dans un espace ? Pourrait-on parler d'un énoncé comme d'une figure idéale et silencieuse ? L'énoncé est toujours donné au travers d'une épaisseur matérielle, même si elle est dissimulée, même si, à peine apparue, elle est condamnée à s'évanouir. (Foucault 1969 : 132)

(33) Le discours suppose le sujet, inscrit prosodiquement, rythmiquement dans le langage, son oralité, sa physique. (Meschonnic 1999 : 74)

De sorte que ce qui semblait pousser O. Ducrot implicitement à remplacer voix par point de vue, à savoir la trop grande matérialité du terme, est exactement ce qui nous incite à le retenir : le dialogisme se manifeste notamment comme hétérogénéité discursive, c'est-à-dire comme interaction d'au moins deux discours. Cette interaction prend des formes linguistiques extrêmement diverses, mais se manifeste de façon identique, par l'impression que l'énoncé dialogique est habité par une / d'autres voix que celle du locuteur-énonciateur $\mathrm{L}_{1} / \mathrm{E}_{1}$ : voix mimée d'un autre locuteur $\mathrm{l}_{1}$; mais aussi, bien plus fréquemment et métaphoriquement, voix attribuée à ce / ces discours avec le(s)quel(s) interagit le discours du locuteur-énonciateur $\mathrm{L}_{1} /$ $\mathrm{E}_{1}$.

Sans faire de voix un terme conceptuel - il n'explique rien -, on le conservera dans l'analyse pour ce qu'il dit de la façon dont les discours sont appréhendés par les sujets : comme faisant entendre, aussi métaphoriquement cela soit-il, une / plusieurs voix, qui signe(nt) la matérialité des discours.

\section{Conclusion}

Notre propos était de questionner la notion de voix dans le champ des études de l'hétérogénéité énonciative: d’abord à sa source, les travaux de Bakhtine; puis dans les approches de la polyphonie et du dialogisme.

Il apparaît que dans les textes bakhtiniens le terme de voix (golos) et ceux qui lui sont associés sont le lieu d'un bourgeonnement où s'entrecroisent les sens physiologico-corporel, discursif et narratologique : rien d'étrange à cela chez un théoricien pour qui le dialogisme est un principe qui, au-delà des discours, irrigue l'homme tout entier, notamment dans sa dimension corporelle.

L’approche polyphonique, dans le cadre pragmatico-énonciatif, a questionné le terme de voix : pour le remplacer, partiellement (O. Ducrot, L. Perrin) ou totalement (la Scapoline), par celui de point de vue, qui, moins incarné, permet de nommer ce qui relèverait d'une instance subjective - l'énonciateur - et ne serait pas lié à la matérialité des mots.

Un tel travail notionnel n'avait pas été fait par l'approche dialogique : nous l'avons entrepris, dans le cadre de l'analyse du discours. En critique de la notion de point de vue qui ne nous a semblé opératoire que pour autant qu'on faisait l'économie de la notion de discours, nous avons tâché de montrer la pertinence du terme de voix: pertinence littérale pour le plan locutoire (que ne prend pas en compte l'approche polyphonique), pertinence métaphorique pour le plan énonciatif: parler de voix, fussent-elles celles de locuteurs-énonciateurs désincarnés comme celles de la sagesse ou de la providence, c'est renvoyer à la dimension corporelle - réelle ou fantasmée - du discours, à sa fondamentale oralité.

\section{Références bibliographiques}


Authier-Revuz J., 1995, Ces mots qui ne vont pas de soi, Paris : Larousse.

Bakhtine M., 1929/1977, Le marxisme et la philosophie du langage, Paris : Minuit.

Bakhtine M., 1934-1935, “Slovo v romane”, in. Voprosy literatury i éstetiki, 72-233, Moscou.

Bakhtine M., 1934/1975/1978, « Du discours romanesque », in Esthétique et théorie du roman, Paris : Gallimard, Tel, 83-233.

Bakhtine M., 1953, “Problema rechevyx rzanrov" in Estetika slovesnogo tvorchestva, 250296, Moscou.

Bakhtine M., 1952/1979/1984a, «Les genres du discours », in Esthétique de la création verbale, Paris : Gallimard, 265-308.

Bakhtine M., 1963, Problemy poetiki Dostoevskogo, Moscou.

Bakhtine M., 1963/1970, Problèmes de la poétique de Dostoïevski, Lausanne: L'âge d'homme.

Bres J., 1998, «Entendre des voix : de quelques marqueurs dialogiques en français », in Bres J., Legrand R., Madray F. et P. Siblot (éd.), L'autre en discours, Montpellier III, Praxiling, 191-212.

Bres J., Haillet P., Mellet S., Nølke H., Rosier L. (éd.), 2005, Dialogisme, polyphonie : approches linguistiques, Actes du colloque de Cerisy, sept 2004.

Bres J. et Nowakowska A., 2006, «Dialogisme : du principe à la matérialité discursive », in Perrin L. (éd.), Le sens et ses voix, Recherches linguistiques 28, Metz : Université de Metz, 21-48.

Bres J. et L. Rosier, 2008, « Réfractions : polyphonie et dialogisme, deux ensembles de reconfigurations théoriques dans les sciences du langage francophones », Slavica Occitania, à paraître.

Bres J. et Verine B., 2003, « Le bruissement des voix dans le discours : dialogisme et discours rapporté », Faits de langue 19, 159-170.

Cahiers de praxématique 43, 2005, Aspects du dialogisme (coord. : A. Nowakowska).

Détrie C., Siblot P. et Verine B., (éd.), 2001, Termes et concepts pour l'analyse du discours.

Une approche praxématique, Paris : Honoré Champion.

Ducrot O., 1980, "Analyse de textes et linguistique de l'énonciation », in Ducrot et al, Les

mots du discours, Paris : Minuit, 7-56.

Ducrot O., 1984, « Esquisse d'une théorie polyphonique de l'énonciation », in Le dire et le dit, Paris, Minuit, p. 171-233.

Ducrot O., 2001, "Quelques raisons de distinguer «locuteurs » et "énonciateurs » ", Polyphonie - linguistique et littéraire, III, 19-41.

Foucault M., 1969, L’archéologie du savoir, Paris : Gallimard.

Meschonnic H., 1999, Poétique du traduire, Verdier.

Moirand S., 2004, «Le dialogisme, entre problématiques énonciatives et théories discursives », Cahiers de praxématique 43, 189-217.

Moirand S., 2007, Les discours de la presse quotidienne, Paris : PUF.

Nølke H., 1994, Linguistique modulaire : de la forme au sens, Louvain Paris : Peeters.

Nølke H., 2006, « Pour une théorie linguistique de la polyphonie », in L. Perrin (éd), 243-269.

Nølke H., Fløttum K., Norén C., 2004, ScaPoLine, La théorie scandinave de la polyphonie linguistique, Paris: Kimé.

Nowakowska A., 2005, «Dialogisme, polyphonie: des textes russes de Bakhtine à la linguistique contemporaine », » in Bres et al. 2005, 19-32.

Perrin L., 2006, « Voix et points de vue dans le discours. De l’opacité linguistique à l’opacité référentielle des expressions », Le Français moderne, 1, 74 ${ }^{\mathrm{e}}$ année, 22-31. 
Perrin L., 2006, (éd.), Le sens et ses voix, Recherches linguistiques 28, Metz : Université Paul Verlaine.

Rosier L., 2006, "Polyphonie : les dessous d'une métaphore », in L. Perrin (éd.), Le sens et ses voix, Recherches linguistiques 28, Metz : Université Paul Verlaine, 189-211. 